\title{
Globular cluster kinematics and X-ray emission in the early-type galaxy NGC 1399
}

\author{
S. Samurovic ${ }^{1,2,3}$ and I. J. Danziger ${ }^{2}$ \\ 1 Dipartimento di Astronomia, Università di Trieste, via Tiepolo 11, 34131 Trieste, Italy \\ e-mail: srdjan@ts.astro.it \\ 2 INAF, Osservatorio Astronomico di Trieste, via Tiepolo 11, 34131 Trieste, Italy \\ e-mail: danziger@ts.astro.it \\ 3 Astronomical Observatory, Volgina 7, 11160 Belgrade, Serbia and Montenegro
}

Received 13 February 2006 / Accepted 7 July 2006

\begin{abstract}
Context. Of the various observational methods used to address the question of dark matter in early-type galaxies there is the possibility of spatial overlap in using kinematics of stars, clusters and X-ray halos.

Aims. To investigate methods for the determination of velocity distribution profiles and deviations from a Gaussian distribution using globular clusters in early-type galaxies, and the implications for dark matter.

Methods. The maximum likelihood method together with standard statistical procedures were used to calculate the velocity dispersion. A new "tracer mass estimator" was applied to obtain a mass estimate based on the globular clusters, which is then compared to the mass obtained using X-rays.

Results. In NGC 1399 the departures from a Gaussian distribution of the velocities at all radial distances are small. Our conclusion is that in spite of the observations that show that the velocity dispersion decreases between 4 and $10 R_{\mathrm{e}}$ there is evidence that dark matter exists beyond $\sim 3 R_{\mathrm{e}}$.
\end{abstract}

Key words. Galaxy: kinematics and dynamics - galaxies: elliptical and lenticular, cD - galaxies: structure - dark matter galaxies: individual: NGC 1399 - Galaxy: star clusters

\section{Introduction}

The presence of dark matter in early-type galaxies continues to be one of the most important unsolved problems in extragalactic astrophysics. Although the currently popular theoretical models (such as cold dark matter models) predict huge amounts of dark matter in these galaxies, some recent observations fail to confirm this for particular galaxies (see, for example, Romanowsky et al. 2003; Peng et al. 2004; Samurović \& Danziger 2005) at least in regions interior to $3-5$ effective radii $\left(R_{\mathrm{e}}\right)$. Some other recent works claim to detect the presence of dark matter in ellipticals at smaller distances from the galactic center (see, for example, Thomas et al. 2005; Teodorescu et al. 2005; Cappellari et al. 2006; De Rijcke et al. 2006). Of special importance are external regions of elliptical galaxies where dark matter might be expected to dominate luminous matter. Studies that use integrated stellar light are limited to 3 to $4 R_{\mathrm{e}}$ because of the low surface brightness beyond these regions. If one wishes to study the mass distribution beyond $\sim 3 R_{\mathrm{e}}$ one can use an X-ray methodology that analyzes hot gas at temperatures $\sim 10^{7} \mathrm{~K}$. Recently, Sivakoff et al. (2004) used the X-ray observations by CHANDRA and assuming hydrostatic equilibrium found that for the X-ray bright galaxy NGC 1600 within $\sim 4 R_{\mathrm{e}}$ dark matter does not dominate. However, two very recent studies by Humphrey et al. (2006) and Fukazawa et al. (2006) used X-ray data to demonstrate the existence of dark matter beyond $\sim 1 R_{\mathrm{e}}$. Some attempts to combine different observational techniques in order to determine the mass of early-type galaxies are given in Kronawitter et al. (2000), Saglia et al. (2000) and Samurović \& Danziger (2005).
Fortunately, beyond $\sim 3 R_{\mathrm{e}}$ one can use other mass tracers such as globular clusters (GCs) and planetary nebulae (PNe) which can be observed at much larger radii. For example, Côté et al. (2003) recently studied NGC 4472 using 263 GCs out to $\sim 570 \operatorname{arcsec}\left(\sim 3.2 R_{\mathrm{e}}\right.$, but this should be $\sim 5.5 R_{\mathrm{e}}$ if they adopted the RC3 value) and found that the radial velocities and density profiles of globular clusters provide "unmistakable evidence" for a massive dark halo. Peng et al. (2004) used PNe in NGC 5128 at distances out to $80 \mathrm{kpc}\left(\sim 15 R_{\mathrm{e}}\right)$ to conclude that dark matter is necessary to explain the observed kinematics, although their value of the mass-to-light ratio (in the $B$-band) $M / L_{B} \sim 13$ is much lower than that commonly thought for early-type galaxies at large radii (Bahcall et al. 1995).

The most important quantity that is extracted from both GCs and PNe radial velocity observations is the velocity dispersion. Frequently authors do not explicitly state how they obtained their estimates of this quantity, so one is led to the conclusion that the adopted velocity distribution is purely Gaussian or that the authors have used a simple statistical definition of the standard deviation to calculate it. In a recent series of papers Dirsch et al. (2003), Richtler et al. (2004) and Dirsch et al. (2004) analyzed the GC system of the galaxy NGC 1399, the central galaxy of the Fornax cluster. They used 468 radial velocities assuming a pure Gaussian distribution to conclude that the velocity dispersion of this galaxy remains approximately constant between 2 and 9 arcmin (corresponding to $12 \mathrm{kpc}$ to $54 \mathrm{kpc}$, which corresponds to approximately 2.86 and $12.86 R_{\mathrm{e}}$, under the assumption that one effective radius is 42 arcsec. This value of the effective radius 
comes from the RC3 catalog (de Vaucoulers et al. 1991) and we verified it using the growth curve of NGC 1399.

In this paper we study the applicability of the assumption of a pure Gaussian distribution function for the GCs using the Dirsch et al. (2004) sample. In Sect. 2 we outline some theoretical aspects relevant to the calculation of the velocity dispersion. In Sect. 3 we present our results for the velocity dispersion based on the sample of Dirsch et al. (2004). In Sect. 4 we compare these results with the results obtained using the X-ray methodology. In Sect. 5 we present mass estimates of NGC 1399 based on globular clusters. Our conclusions are presented in Sect. 6.

\section{Mathematical background}

When dealing with the velocities related to the GCs and/or PNe one usually assumes a pure Gaussian velocity distribution: for example, Grillmair et al. (1994) analyzed 47 GCs within 9 arcmin in NGC 1399 using the methodology developed by Morrison et al. (1990) to calculate a velocity dispersion $\left(\sigma=388 \pm 54 \mathrm{~km} \mathrm{~s}^{-1}\right)$ and a mass-to-light ratio in the $B$-band $\left(M / L_{B}=79 \pm 20\right.$, interior to 9 arcmin $)$. This technique uses a maximum likelihood approach and Morrison et al. state that since this technique is model-dependent, slightly nonGaussian distributions can have significant effects on the performance of estimators. Richtler et al. (2004) used the maximum likelihood dispersion estimator given in Pryor \& Meylan (1993). The Gaussian estimator can be expressed as:

$f_{\mathrm{G}}\left(v_{i}\right)=\frac{1}{\sqrt{2 \pi\left(\sigma^{2}+\delta_{i}^{2}\right)}} \exp \left(-\frac{\left(v_{i}-\bar{v}\right)^{2}}{2\left(\sigma^{2}+\delta_{i}^{2}\right)}\right)$

where $\sigma$ is the velocity dispersion, and it is assumed that each velocity $v_{i}$ (the GC velocity from which the systemic velocity, $v_{\text {sys }}$, is subtracted-in the case of NGC $1399 v_{\text {sys }}$ is fixed to $1441 \mathrm{~km} \mathrm{~s}^{-1}$ ) is drawn from a normal, Gaussian, distribution. $\bar{v}$ is the mean velocity and $\delta_{i}$ is a known measurement uncertainty of $v_{i}$. This equation is valid only if the distribution of errors is Gaussian.

The maximum likelihood function is then given as

$L_{\mathrm{G}}=\prod_{i} \frac{1}{\sqrt{2 \pi\left(\sigma^{2}+\delta_{i}^{2}\right)}} \exp \left(-\frac{\left(v_{i}-\bar{v}\right)^{2}}{2\left(\sigma^{2}+\delta_{i}^{2}\right)}\right)$.

Taking the logarithm of Eq. (2) and finding

$$
\frac{\partial \ln L_{\mathrm{G}}}{\partial \sigma}=0
$$

one can calculate the velocity dispersion using an iterative procedure as:

$$
\sum_{i} \frac{\left(v_{i}-\bar{v}\right)^{2}}{\left(\sigma^{2}+\delta_{i}^{2}\right)^{2}}=\sum_{i} \frac{1}{\sigma^{2}+\delta_{i}^{2}}
$$

where the sum includes all the velocities in a given bin. In all our calculations below we always set $\bar{v}=0$. Using expressions given in Pryor \& Meylan (1993) one can then calculate the uncertainty of the resulting velocity dispersion (these data were presented in Table 2 of Richtler et al. (2004) for different bins and different selections).

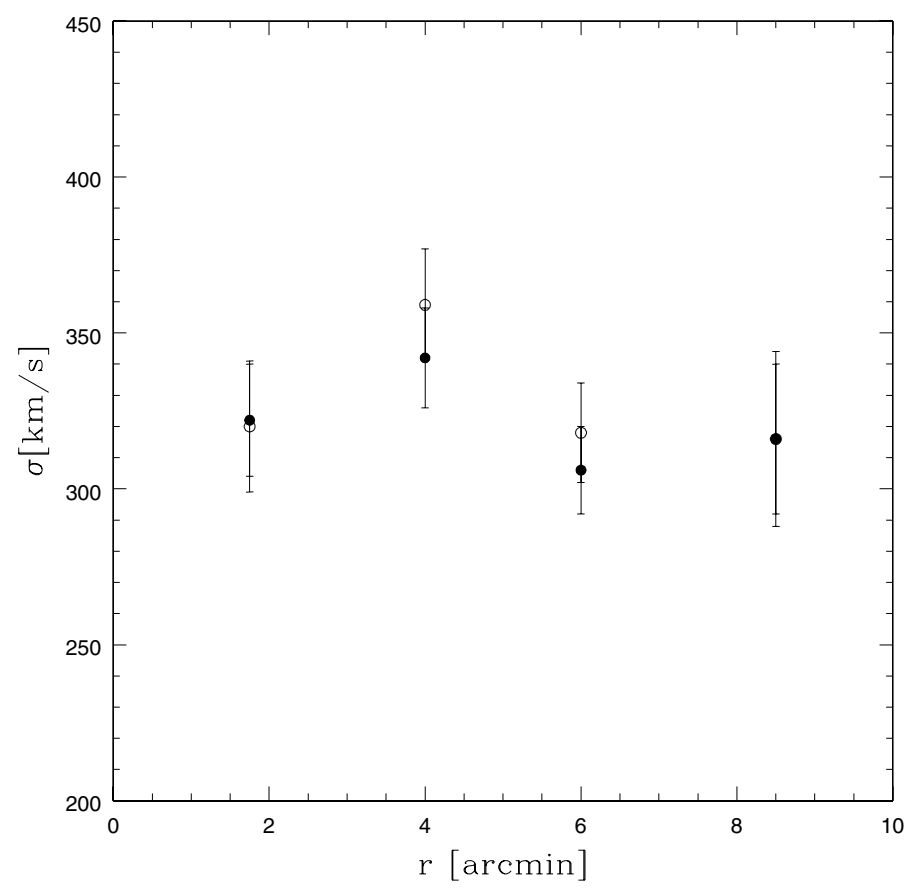

Fig. 1. Comparison between our calculation of the velocity dispersion of NGC 1399 (open circles) and calculations of Richtler et al. (2004) (filled circles), when a pure Gaussian distribution is assumed. Note that for the outermost point the two values overlap.

\section{Globular clusters of NGC 1399}

The globular cluster system of NGC 1399 was presented in Dirsch et al. (2003, wide-field photometry), Richtler et al. (2004, kinematics) and Dirsch et al. (2004, spectroscopy and database). In our analysis, we use the set of 445 GCs for which the estimated uncertainties are $\delta_{i}<60 \mathrm{~km} \mathrm{~s}^{-1}$. This selection enabled us to have a sufficiently large number of clusters in each chosen radial bin and it was also useful in the construction of the bins given in Table 2, which contain approximately 50 clusters each. We took as a center of NGC 1399 the coordinates (J2000.0) given in the NED database: $\alpha=03^{\mathrm{h}} 38^{\mathrm{m}} 29.08$ and $\delta=-35^{\circ} 27^{\prime} 02^{\prime \prime}$. 7 . In Fig. 1 we present the comparison of our results (open circles) with those of Richtler et al. (2004) (filled circles; the second column of their Table 2, no selection was performed). In this case a pure Gaussian distribution is assumed and the agreement is very good. In Fig. 2 we present the comparison of our results (open symbols) with those of Richtler et al. (2004) (filled symbols) for red $(C-R>1.6)$ and blue $(C-R<1.6)$ clusters using the Richtler et al. bins (see also Table 1) (where the data for Washington $C$ and Kron-Cousins $R$ are taken from Dirsch et al. 2004). Our total number of red and blue clusters used in this comparison is 412: we have taken into account all the clusters for which we had colours and for which $\delta_{i}<60 \mathrm{~km} \mathrm{~s}^{-1}$. A pure Gaussian distribution is assumed. If we place all GCs of a given colour in one bin our results are in very good agreement with those of Richtler et al:: for red GCs we found $\sigma_{\text {red }}=$ $259 \pm 11 \mathrm{~km} \mathrm{~s}^{-1}$ and they found $\sigma_{\text {red }}=255 \pm 13 \mathrm{~km} \mathrm{~s}^{-1}$, and for blue GCs we found $\sigma_{\text {blue }}=295 \pm 12 \mathrm{~km} \mathrm{~s}^{-1}$ and they found $\sigma_{\text {blue }}=291 \pm 14 \mathrm{~km} \mathrm{~s}^{-1}$. However, there are discrepancies related to the individual bins for the velocity dispersion values of Richtler et al. which we may attribute to our selection criterion: we took only GCs for which $\delta_{i}<60 \mathrm{~km} \mathrm{~s}^{-1}$.

In Table 1 we list our results for the projected velocity dispersion in the case of the pure Gaussian velocity distribution (with $\delta_{i}<60 \mathrm{~km} \mathrm{~s}^{-1}$ ) for the total sample of clusters. In this table 
Table 1. Projected velocity dispersion measurements of NGC 1399 for a Gaussian distribution $\left(\delta_{i}<60 \mathrm{~km} \mathrm{~s}^{-1}\right)$.

\begin{tabular}{|c|c|c|c|c|c|c|c|}
\hline $\begin{array}{l}\text { Radial bin } \\
\text { (arcmin) } \\
(1)\end{array}$ & $\begin{array}{c}\langle r\rangle \\
(\operatorname{arcmin}) \\
(2)\end{array}$ & $\begin{array}{c}\sigma_{\text {tot }} \\
\left(\mathrm{km} \mathrm{s}^{-1}\right) \\
(3)\end{array}$ & $N_{\text {tot }}$ & $\begin{array}{c}\sigma_{\text {blue }} \\
\left(\mathrm{km} \mathrm{s}^{-1}\right) \\
(5)\end{array}$ & $N_{\text {blue }}$ & $\begin{array}{c}\sigma_{\text {red }} \\
\left(\mathrm{km} \mathrm{s}^{-1}\right) \\
(7)\end{array}$ & $N_{\text {red }}$ \\
\hline All $r$ & - & $331 \pm 11$ & 445 & $295 \pm 12$ & 196 & $259 \pm 11$ & 216 \\
\hline$r<3.5$ & 1.75 & $0 \pm 21$ & 124 & $223 \pm$ & 51 & $=15$ & 66 \\
\hline $2.5<r<5.5$ & 4.00 & $359 \pm 18$ & 201 & $265 \pm 14$ & 80 & $255 \pm 13$ & 107 \\
\hline $4.5<r<7.5$ & 6.00 & $15 \pm$ & 209 & $266 \pm$ & 90 & $230 \pm 12$ & 103 \\
\hline$r>6.5$ & 8.50 & $316 \pm 28$ & 127 & $248 \pm 14$ & 67 & $243 \pm 16$ & 53 \\
\hline
\end{tabular}

Notes: Column (1): radial bin; Col. (2): central point for a given bin; Col. (3): velocity dispersion with the estimated uncertainty for a total sample of clusters; Col. (4): number of objects in a given bin for a total sample of clusters; Col. (5): velocity dispersion with the estimated uncertainty for blue clusters; Col. (6): number of objects in a given bin for blue clusters; Col. (7): velocity dispersion with the estimated uncertainty for red clusters; Col. (8): number of objects in a given bin for red clusters.

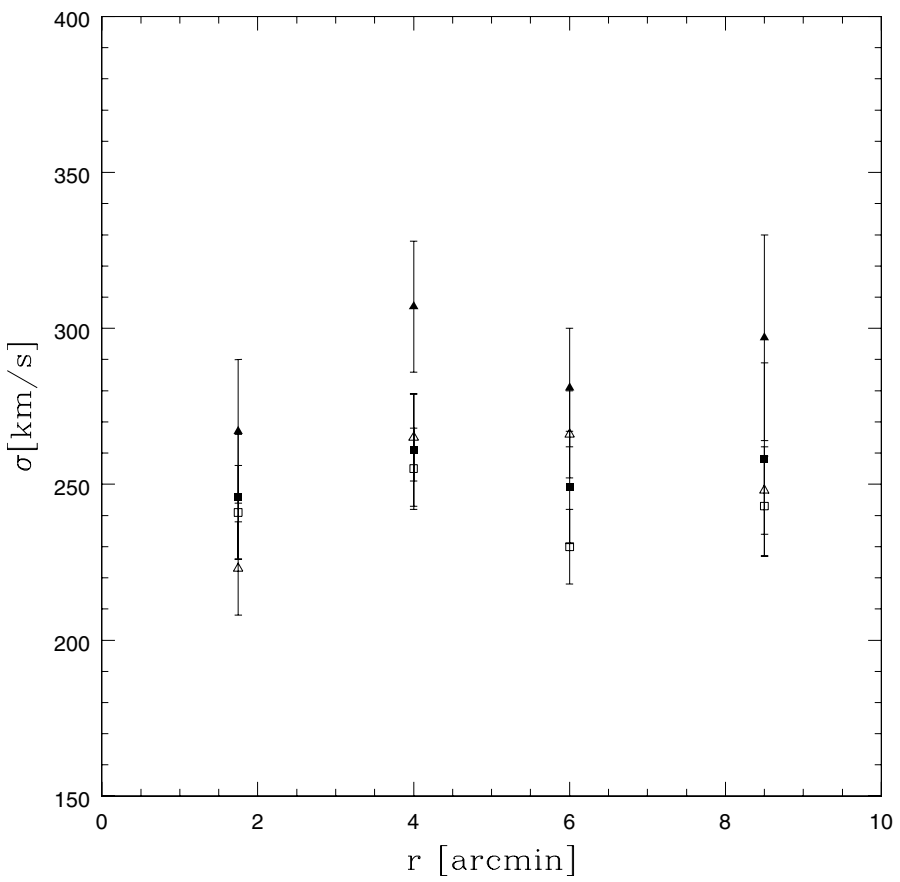

Fig. 2. Comparison between our calculation of the velocity dispersion of NGC 1399 for red (open rectangles) and blue clusters (open triangles) and calculations of Richtler et al. (2004) for red (filled rectangles) and blue clusters (filled triangles). In all the cases we assumed a pure Gaussian distribution.

we also give our results for blue and red clusters. We have used the bins defined in the Richtler et al. (2004) paper: as can be seen from Fig. 1 with these bins the velocity dispersion remains approximately constant throughout the galaxy. Errors were calculated using the lengthy expressions of Pryor \& Meylan (1993). With Richtler et al. binning of the data there exists a significant overlap of the bins: that is, there are many of the same clusters present in two different bins. Therefore, we bin the data taking approximately 50 GCs in each separate bin (see Table 2): using this approach and assuming a Gaussian distribution ${ }^{1}$, we see a decreasing trend in the velocity dispersion between $\sim 3$ and $\sim 7$ arcmin (see also Fig. 3; more details are given below in this section). For example, using large (Richtler et al.) bins the last

\footnotetext{
${ }^{1}$ We checked the projected velocity dispersion for the cases of departure from a pure Gaussian velocity distribution using $s_{3}$ and $s_{4}$ parameters presented in Table 2 and we did not find large differences: the calculated values thus obtained are equal to the ones obtained using a pure Gaussian within the error bars.
}

measured point was calculated for all GCs beyond 6.5 arcmin and some clusters (73 clusters between 6.5 and 7.5 arcmin) were already taken into account in a preceding bin. For all the given bins we calculated the velocity dispersion and the skewness and kurtosis parameters, $s_{3}$ and $s_{4}$, using standard definitions and the NAG routine GO1AAF (see Table 2 in which we present the results based on the maximum likelihood function and, for comparison, the result obtained using the standard statistical definition). We are not trying to reconstruct the full line-of-sight velocity distribution, because it is known (see Merritt 1997) that for small samples such as ours which contain less than a few hundred objects per bin it is not possible - we simply calculate skewness and kurtosis parameters (we do not attribute much of significance to the numerical values of these parameters) in order to determine whether in some bin a significant departure from a Gaussian distribution exists. This is similar to the approach applied by Teodorescu et al. (2005) in their Fig. 18. The results are graphically presented in Fig. 3 (left). The results based on the maximum likelihood function and those based on the standard statistical method are in very good agreement. In order to test whether differences in binning affect our calculations we also performed the calculations using different binning with variable numbers of the GCs within a bin of fixed length ( 2 arcmin) without overlap of the bins. In Tables 3 and 4 we present the results for the blue and red clusters, respectively. Here we have used a bin width of 2 arcmin in order to include in each bin a statistically significant number of clusters. In Tables 5 and 6 we present two different types of binnings for which the center of each bin is placed on odd and even values of the radius, respectively (the obvious exceptions are the first and the last bin in Table 6). Table 7 presents the results of one additional binning check for which each bin contains approximately 75 clusters (instead of 50). The results for the blue, red and the total sample (which includes both blue and red clusters) given in Tables 3-7 were obtained using the same standard statistical procedure based on the same NAG routine; they are graphically presented in Figs. 3 (right) and 4. Although in Fig. 3 (left) we can see a declining trend in the velocity dispersion, the reduced $\chi^{2}$ value is low for a constant velocity dispersion of $330 \mathrm{~km} \mathrm{~s}^{-1}$ and is equal to 1.1 (the best fit case, when the first point is excluded). The constant value of the velocity dispersion that ranges between 320 and $330 \mathrm{~km} \mathrm{~s}^{-1}$ provides the best fit case when $N=75$ (the right hand panel of Fig. 3), but then $\chi^{2} \approx 1.3$ (all points are included in the fit).

For the total sample of clusters (Fig. 3) we see a low velocity dispersion in the inner region (interior to $\sim 2$ arcmin), a higher value of the velocity dispersion (beyond $\sim 2$ arcmin) and a decrease of the velocity dispersion between $\sim 3$ and $\sim 7$ arcmin (more evident in the left panel of Fig. 3, because of the binning 
Table 2. Projected velocity dispersion measurements of NGC 1399 for a Gaussian distribution with a fixed number of clusters per bin $\left(\delta_{i}<\right.$ $\left.60 \mathrm{~km} \mathrm{~s}^{-1}, N \approx 50\right)$.

\begin{tabular}{ccccccccccc}
\hline \hline $\begin{array}{c}\text { Radial bin } \\
(\operatorname{arcmin}) \\
(1)\end{array}$ & $\begin{array}{c}\langle r\rangle \\
(\operatorname{arcmin})\end{array}$ & $\begin{array}{c}\sigma_{\mathrm{ML}} \\
\left(\mathrm{km} \mathrm{s}^{-1}\right)\end{array}$ & $\begin{array}{c}\text { err_o } \sigma_{\mathrm{ML}} \\
(3)\end{array}$ & $\begin{array}{c}\sigma_{\text {stat }} \\
(4)\end{array}$ & $\begin{array}{c}\text { err_ } \sigma_{\text {stat }} \\
\left(\mathrm{km} \mathrm{s}^{-1}\right) \\
(5)\end{array}$ & $\begin{array}{c}\mathrm{km} \mathrm{s}_{3} \\
(6)\end{array}$ & $\begin{array}{c}\text { err_s } s_{3} \\
(7)\end{array}$ & $s_{4}$ & err_s $s_{4}$ & $N$ \\
\hline $0.000<r<2.543$ & 1.27 & 230 & 24 & 234 & 23 & 0.54 & 0.35 & 0.74 & 0.48 & 50 \\
$2.543 \leq r<3.240$ & 2.89 & 398 & 41 & 396 & 40 & -0.07 & 0.35 & -0.23 & 0.48 & 50 \\
$3.240 \leq r<3.860$ & 3.55 & 379 & 40 & 373 & 37 & 0.90 & 0.35 & 3.51 & 0.48 & 50 \\
$3.860 \leq r<4.880$ & 4.37 & 334 & 34 & 338 & 34 & -0.55 & 0.35 & 0.41 & 0.48 & 50 \\
$4.880 \leq r<5.528$ & 5.20 & 342 & 36 & 346 & 35 & -0.21 & 0.35 & 0.21 & 0.48 & 50 \\
$5.528 \leq r<6.214$ & 5.87 & 303 & 31 & 307 & 30 & 0.83 & 0.34 & 2.17 & 0.47 & 51 \\
$6.214 \leq r<6.950$ & 6.58 & 291 & 30 & 297 & 30 & -0.60 & 0.35 & 0.02 & 0.48 & 50 \\
$6.950 \leq r<7.690$ & 7.32 & 304 & 33 & 301 & 30 & 0.17 & 0.35 & 1.42 & 0.49 & 49 \\
$7.690 \leq r<10.380$ & 9.04 & 352 & 38 & 358 & 38 & -0.03 & 0.37 & -0.80 & 0.53 & 45 \\
\hline
\end{tabular}

Notes: Column (1): radial bin; Col. (2): central point for a given bin; Col. (3): velocity dispersion for a Gaussian distribution; Col. (4): formal errors for the velocity dispersion; Col. (5): velocity dispersion obtained using the standard statistical definition; Col. (6): formal errors for the velocity dispersion obtained using standard statistical definitions; Col. (7): $s_{3}$ parameter; Col. (8): formal errors for the $s_{3}$ parameter; Col. (9): $s_{4}$ parameter; Col. (10): formal errors for the $s_{4}$ parameter; Col. (11): number of clusters in a given bin.

applied). Beyond $\sim 8$ arcmin we notice an increase in the velocity dispersion. We confirm the finding of Richtler et al. (2004) regarding the innermost point $(<2$ arcmin). Beyond $\sim 2$ arcmin our results differ somewhat from those by Richtler et al. because of the binning and selection criteria used.

The red clusters show a lower velocity dispersion than the blue clusters (see Fig. 4) and for a possible explanation we refer the reader to Sect. 4 of the paper by Richtler et al. (2004). Although both $s_{3}$ and $s_{4}$ parameters are small we see that while the calculated $s_{3}$ parameters for blue clusters differ from those for red clusters, the $s_{4}$ parameters for both classes are very similar. The binning in both cases was such that we kept the bin width constant and equal to 2 arcmin in order to have enough clusters per bin.

Forte et al. (2005) state there are indications (albeit not conclusive) that there could be an association between the red GCs and the stellar component and that blue GCs show a spatial distribution which is similar to that inferred for dark matter. However any attempt to draw conclusions from this is outside the province of this paper.

As an additional test we also calculated the velocity dispersion in the inner part of NGC 1399 (interior to $\sim 2$ arcmin) in which we have 14 GCs (the innermost at $\sim 1.3$ arcmin and the outermost at $\sim 2$ arcmin). We calculated that for the total sample of blue and red clusters the velocity dispersion is $232 \pm 62 \mathrm{~km} \mathrm{~s}^{-1}$. We note that this result is in agreement with the integrated stellar light observations thus giving credence to the feasibility of calculating galaxy kinematics even with the small number of mass tracers in a given bin.

\section{X-ray modelling}

The X-ray methodology of studying mass in early-type galaxies is well known (see e.g. Mathews \& Brighenti 2003; Samurović $\&$ Danziger 2005). We assume that spherical symmetry in the galaxy holds, and that the condition of hydrostatic equilibrium is valid. For clarity we adopt the following 3 formulae from the referenced papers:

1) the total mass of the galaxy interior to the radius $r$ is calculated using the following equation taken from Kim \& Fabbiano (1995):

$$
M_{T}=1.8 \times 10^{12}(3 \beta+\alpha)\left(\frac{T}{1 \mathrm{keV}}\right)\left(\frac{r}{10^{3 \prime \prime}}\right)\left(\frac{d}{10 \mathrm{Mpc}}\right) M_{\odot},(5)
$$

where the exponent $\alpha$ is related to the temperature $\left(T \sim r^{-\alpha}\right)$ and in our calculations is taken to be zero, and $\beta$ is the slope used in the analytic King approximation model and is taken to be $\beta=0.35$ (Jones et al. 1997) and $\beta=0.50$ (interior to 50 arcsec, see Paolillo et al. 2002);

2) the mass-to-light ratio in the $B$-band is calculated as a function of radius $r$ :

$$
\begin{aligned}
\frac{M_{T}}{L_{B}}= & 1.16 \times 10^{-2} 10^{\frac{B}{2.5}}(3 \beta+\alpha) \\
& \times\left(\frac{T}{1 \mathrm{keV}}\right)\left(\frac{r}{10^{3 \prime \prime}}\right)\left(\frac{d}{10 \mathrm{Mpc}}\right)^{-1},
\end{aligned}
$$

where $B$ is the $B$ magnitude of the galaxy inside radius $r$ (Kim \& Fabbiano 1995);

3) the following simple formula taken from Mathews \& Brighenti (2003) was used as an estimate of the temperature based on the stellar velocity dispersion assuming a virial condition:

$$
k T_{\sigma[\mathrm{keV}]}=6.367 \times 10^{-6} \sigma_{\left[\mathrm{km} \mathrm{s}^{-1}\right]}^{2}
$$

where the temperature, $T_{\sigma}$, is given in $\mathrm{keV}$ and the stellar velocity dispersion, $\sigma$, is given in $\mathrm{km} \mathrm{s}^{-1}$.

In Fig. 5 we show the mass-to-light ratio of the galaxy NGC 1399 in the $B$-band using Eq. (6). The parameter $\beta$ was taken to be 0.35 (dashed strip) and 0.50 (dotted strip) as given above. We present the estimates for the mass-to-light ratio beyond one effective radius; this limit is taken because we want to avoid the problems due to cooling flows in the central region and because we are interested in the comparison of different methodologies in the outer regions of the galaxies where dark matter is expected to play a significant role. For the discussion about the reliability of the X-ray mass estimates in the regions of the cooling flow and the problems related to the deviations from hydrostatic equilibrium see the paper by Ciotti \& Pellegrini (2004). The sample of X-ray galaxies from O'Sullivan et al. (2003), which also includes NGC 1399, seems to show that beyond $\sim 1 R_{\mathrm{e}}$, for the set of galaxies with a cooling core (to which NGC 1399 belongs) there is not much cooling. Since in our calculations we always assume a constant temperature in a given region, as stated above $(\alpha=0)$, in Fig. 5 we present 

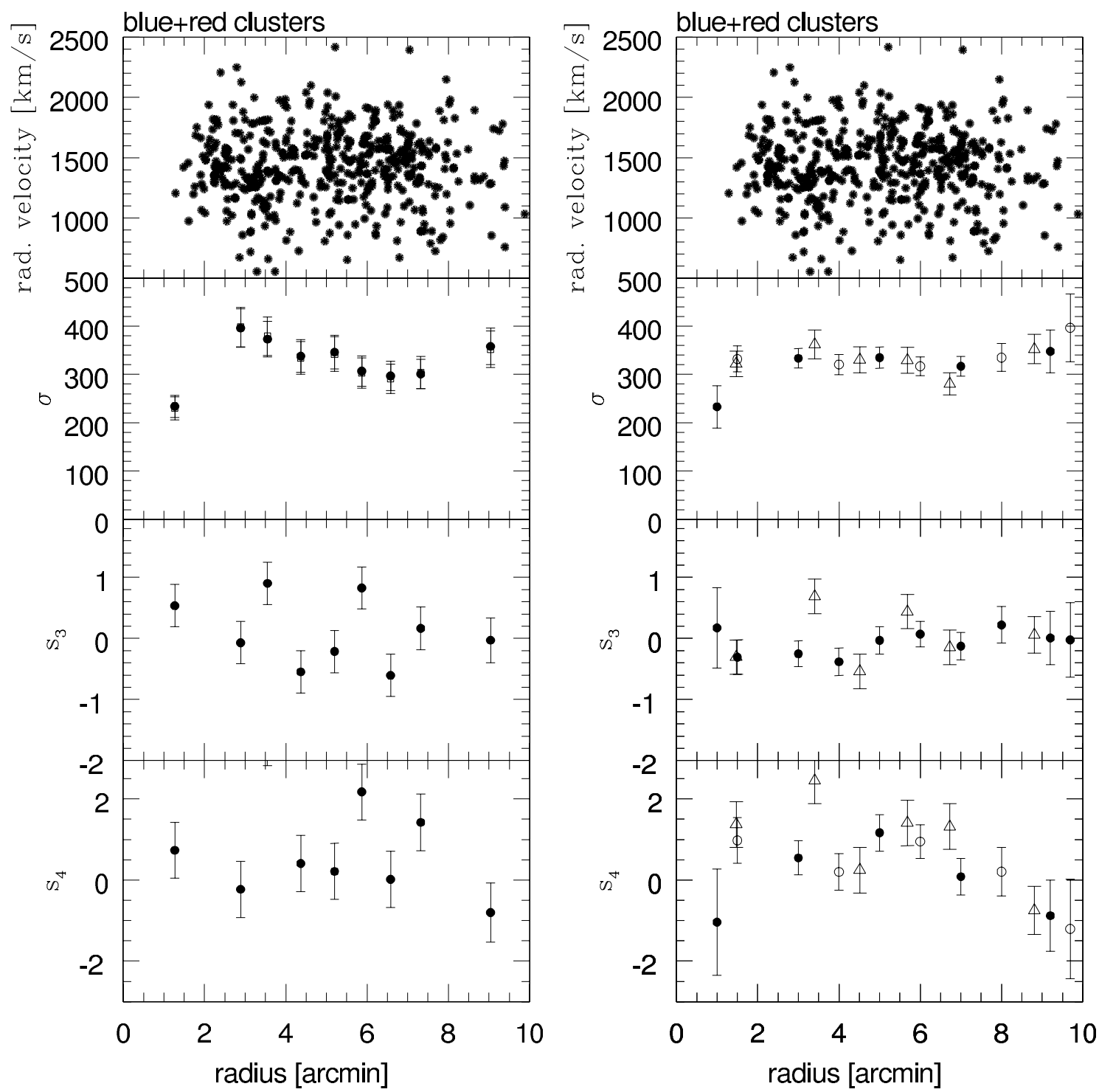

Fig. 3. Kinematics of NGC 1399 based on the total sample of red and blue GCs. Left: from top to bottom: radial velocity of the GCs in km $\mathrm{s}^{-1}$, velocity dispersion calculated in a given bin using a maximum likelihood method (open squares) and using standard statistical procedures (filled circles), $s_{3}$ and $s_{4}$ parameters. In all the bins the total number of clusters per bin was approximately 50. Right: the meaning of the data on the ordinate is the same as in the left panel. It combines results for binning methods given as follows: filled circles represent the data in Table 5, open circles are for the data given in Table 6 and open triangles are for the data from Table 7.

estimates of the mass-to-light ratio in three different regions characterized by different temperatures:

(i) interior to 100 arcsec we used Eq. (7) and allowed the interval for the temperature between $T_{\sigma}$ and $1.5 T_{\sigma}$ (virial assumption). The temperature was taken to be $0.61 \mathrm{keV}$ as given in Brown \& Bregman (1998) signifying that the value of the velocity dispersion taken was $\sim 310 \mathrm{~km} \mathrm{~s}^{-1}$. We see that within $100 \operatorname{arcsec}\left(\sim 2.4 R_{\mathrm{e}}\right)$ for $\beta=0.35$ dark matter does not dominate, because the maximum value that the mass-to-light ratio can reach is $\sim 10$. The same is true for the lower value of the temperature $(T=0.61 \mathrm{keV})$ and $\beta=0.50$, whereas for the higher values of the temperature and $\beta=0.50$ the mass-to-light ratio increases suggesting the existence of dark matter (in this case the mass-tolight ratio can reach values of $\sim 15$. The lack of dark matter within $\sim 2.4 R_{\mathrm{e}}$ is consistent with our results for IC 1459 , IC 3370 (Samurović and Danziger 2005): for these two galaxies we did not find evidence for dominance of dark matter closer than $\sim 3 R_{\mathrm{e}}$. Saglia et al. (2000), using integrated stellar light, found that $M / L_{B}=10$ (inside $\sim 1.5 R_{\mathrm{e}}$, for the distance of $17.6 \mathrm{Mpc}$; this scales to $\sim 9$ for the distance of $20.36 \mathrm{Mpc}$ used in our paper) and at $\sim 2 R_{\mathrm{e}}$ they found that $M / L_{B} \sim 12-15$ which scales to $\sim 10.4-13$ for our adopted distance. It is also consistent with the results obtained by Méndez et al. (2001) who studied 535 PNe in the flattened elliptical galaxy NGC 4697 and found that, assuming isotropic velocity distribution, this galaxy does not show dark matter within $3 R_{\mathrm{e}}$;

(ii) between 100 and $150 \operatorname{arcsec}$ we used as a lower value $T \sim$ $0.88 \mathrm{keV}$ and as an upper value $T \sim 1.2 \mathrm{keV}$ estimated from the paper of Jones et al. (1997) (their Fig. 3);

(iii) beyond $150 \operatorname{arcsec}$ we used $T=1.30 \pm 0.05$ estimated by Jones et al. (1997).

For comparison, in Fig. 5 we have also plotted the Grillmair et al. (1994) value which we transformed from the value of $79 \pm 20$ at $13.2 \mathrm{kpc}$ to $51 \pm 13$ at the distance of $20.36 \mathrm{Mpc}$ used in our 
Table 3. Kinematics data for NGC 1399 for the blue clusters.

\begin{tabular}{cccccccc}
\hline \hline $\begin{array}{c}\langle r\rangle \\
(\operatorname{arcmin})\end{array}$ & $\begin{array}{c}\sigma_{\text {blue }} \\
\left(\mathrm{km} \mathrm{s}^{-1}\right)\end{array}$ & $\begin{array}{c}\text { err_o } \sigma_{\text {blue }} \\
\left(\mathrm{km} \mathrm{s}^{-1}\right)\end{array}$ & $s_{\text {3blue }}$ & err_s $s_{\text {3blue }}$ & $s_{\text {4blue }}$ & err_s $s_{\text {blue }}$ & $N$ \\
\hline 1 & $(2)$ & $(3)$ & $(4)$ & $(5)$ & $(6)$ & $(7)$ & $(8)$ \\
\hline 3 & 185 & 59 & 0.14 & 1.10 & -1.60 & 4.80 & 5 \\
5 & 352 & 33 & -0.07 & 0.32 & 0.20 & 0.41 & 58 \\
7 & 389 & 38 & 0.35 & 0.33 & 0.60 & 0.44 & 54 \\
9.2 & 344 & 31 & -0.07 & 0.32 & 0.00 & 0.40 & 60 \\
\hline
\end{tabular}

Notes: Column (1): central point for a given bin; Col. (2): velocity dispersion for the blue clusters; Col. (3): formal errors for the velocity dispersion of the blue clusters; Col. (4): $s_{3}$ parameter for the blue clusters; Col. (5): formal errors for the $s_{3}$ parameter for the blue clusters; Col. (6): $s_{4}$ parameter for the blue clusters; Col. (7): formal errors for the $s_{4}$ parameter for the blue clusters; Col. (8): number of clusters in a given bin. In all the cases $\delta_{i}<60 \mathrm{~km} \mathrm{~s}^{-1}$.

Table 4. Kinematics data for NGC 1399 for the red clusters.

\begin{tabular}{cccccccc}
\hline \hline $\begin{array}{c}\langle r\rangle \\
\left(\begin{array}{c}\operatorname{arcmin}) \\
(1)\end{array}\right.\end{array}$ & $\begin{array}{c}\sigma_{\text {red }} \\
\left(\mathrm{km} \mathrm{s}^{-1}\right) \\
(2)\end{array}$ & $\begin{array}{c}\text { err_o } \sigma_{\text {red }} \\
\left(\mathrm{km} \mathrm{s}^{-1}\right)\end{array}$ & $s_{3 \text { red }}$ & err_s $s_{3 \text { red }}$ & $s_{4 \text { red }}$ & err_ $s_{4 \text { red }}$ & $N$ \\
\hline 1 & 223 & 56 & $(4)$ & $(5)$ & $(6)$ & $(7)$ & $(8)$ \\
3 & 325 & 27 & -0.42 & 0.87 & -0.80 & 3.00 & 8 \\
5 & 286 & 26 & -0.56 & 0.31 & 1.60 & 0.39 & 61 \\
7 & 284 & 26 & -0.42 & 0.32 & -0.40 & 0.40 & 60 \\
9.2 & 275 & 56 & 0.49 & 0.71 & -0.60 & 2.00 & 12 \\
\hline
\end{tabular}

Notes: The same as in Table 3 but for the red clusters.

Table 5. Kinematics data for NGC 1399 for the total sample of blue and red clusters (part 1).

\begin{tabular}{cccccccc}
\hline \hline $\begin{array}{c}\langle r\rangle \\
(\operatorname{arcmin})\end{array}$ & $\begin{array}{c}\sigma_{\text {total1 }} \\
\left(\mathrm{km} \mathrm{s}^{-1}\right)\end{array}$ & $\begin{array}{c}\text { err_o } \sigma_{\text {total1 }} \\
\left(\mathrm{km} \mathrm{s}^{-1}\right)\end{array}$ & $s_{3 \text { total1 }}$ & err_s $s_{3 \text { total1 }}$ & $s_{4 \text { total1 }}$ & err_s $s_{4 \text { total1 }}$ & $N$ \\
\hline 1 & $(2)$ & $(3)$ & $(4)$ & $(5)$ & $(6)$ & $(7)$ & $(8)$ \\
3 & 233 & 44 & 0.14 & 0.65 & -1.00 & 1.71 & 14 \\
5 & 334 & 20 & -0.28 & 0.21 & 0.60 & 0.18 & 135 \\
7 & 335 & 22 & 0.00 & 0.23 & 1.20 & 0.21 & 117 \\
9.2 & 317 & 20 & -0.14 & 0.22 & 0.00 & 0.20 & 120 \\
\hline
\end{tabular}

Notes: The same as in Table 3 but for the total sample of blue and red clusters (bins centered on odd values of radius, see text for details).

paper (see Fig. 3, left). We will address this problem later in this section and also in the next section.

Although the X-ray data suggest that beyond $\sim 3 R_{\mathrm{e}}$ in NGC 1399 there should be a significant amount of dark matter, we note, however, that since NGC 1399 is a central galaxy of the Fornax cluster, the confinement of the hot gas may be assisted by the external pressure of the intercluster medium (ICM) and is not wholly due to the gravitational field of NGC 1399 (Bertin 2000).

\section{Mass estimates based on globular clusters}

Recently Evans et al. (2003) introduced a new "tracer mass estimator" which provides an estimate of the enclosed mass from the projected positions and line-of-sight velocities of a given tracer population (such as GCs and PNe). One can assume that the tracer population is spherically symmetric and has a number density that obeys a power law:

$\rho(r)=\rho_{0}\left(\frac{a}{r}\right)^{\gamma}$

where $a$ is constant, and radius $r$ ranges between $r_{\text {in }}$ and $r_{\text {out }}$, inner and outer points of the given population, respectively. The parameter $\gamma$ is determined using the surface density of the tracer population between $r_{\text {in }}$ and $r_{\text {out }}$. Evans et al. give the following formula for the mass enclosed within $r_{\text {in }}$ and $r_{\text {out }}$ for the isotropic ("iso" in the formulas below) case ("los" stands for line-of-sight):

$M=\frac{C_{\text {iso }}}{G N} \sum_{i} v_{\operatorname{los} i}^{2} R_{i}$,

where constant $C_{\text {iso }}$ is given as:

$C_{\text {iso }}=\frac{4(\alpha+\gamma)}{\pi} \frac{4-\alpha-\gamma}{3-\gamma} \frac{1-\left(r_{\text {in }} / r_{\text {out }}\right)^{3-\gamma}}{1-\left(r_{\text {in }} / r_{\text {out }}\right)^{4-\alpha-\gamma}}$.

The parameter $\alpha$ for an isothermal potential is equal to zero and this value was taken in all the calculations that we performed below. This is a reasonable assumption (see Evans et al. 2003, for details). We are aware of the initial assumptions about the isothermal potential which predicts a quasi-constant velocity dispersion profile but the calculated value of the mass is not much influenced by this. This assumption was used by Peng et al. (2004) in the calculation of the total mass of NGC 5128 for which there is a declining trend of the velocity dispersion.

For the application of Eq. (10) we split the total sample of GCs into 5 bins: each bin (except the first one which is 2 arcmin wide) contains the previous one in addition to a fixed width of 

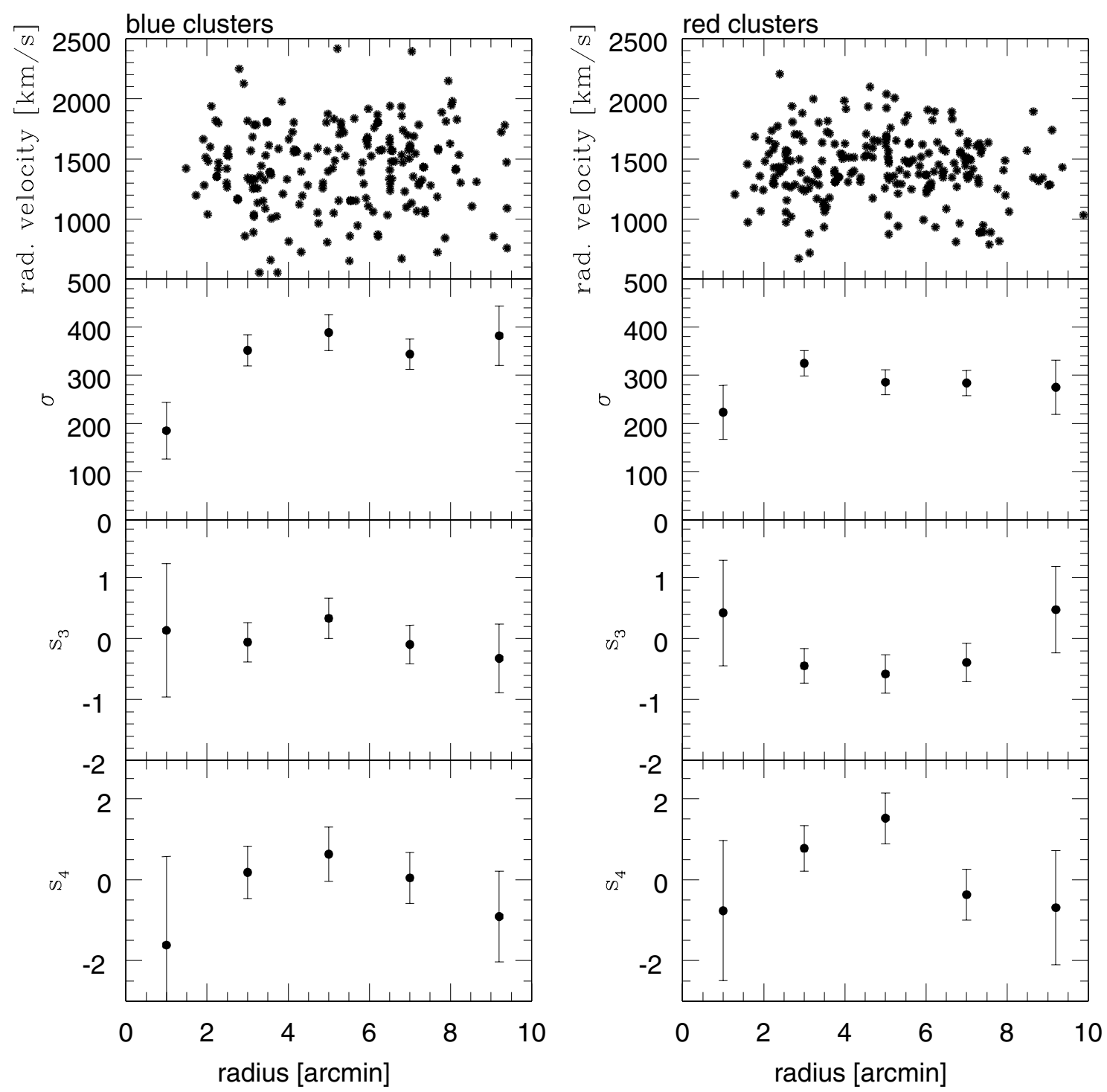

Fig. 4. Kinematics of NGC 1399 based on the total sample of blue (left) and red (right) GCs. From top to bottom: radial velocity of the GCs in $\mathrm{km} \mathrm{s}^{-1}$, velocity dispersion, $s_{3}$ and $s_{4}$ parameters calculated in a given bin from Tables 3 and 4 .

Table 6. Kinematics data for NGC 1399 for the total sample of blue and red clusters (part 2).

\begin{tabular}{|c|c|c|c|c|c|c|c|}
\hline$\langle r\rangle$ & $\sigma_{\text {total2 }}$ & err_ $\sigma_{\text {total2 }}$ & $s_{3 \text { total2 }}$ & err__ $s_{3 \text { total } 2}$ & $s_{4 \text { total2 }}$ & err_s $s_{4 \text { total2 }}$ & $N$ \\
\hline $\begin{array}{c}(\operatorname{arcmin}) \\
(1)\end{array}$ & $\begin{array}{c}\left(\mathrm{km} \mathrm{s}^{-1}\right) \\
(2)\end{array}$ & $\begin{array}{c}\left(\mathrm{km} \mathrm{s}^{-1}\right) \\
(3)\end{array}$ & (4) & (5) & (6) & (7) & (8) \\
\hline 1.5 & 332 & 27 & -0.28 & 0.28 & 1.00 & 0.32 & 76 \\
\hline 4 & 321 & 21 & -0.42 & 0.22 & 0.20 & 0.20 & 119 \\
\hline 6 & 317 & 19 & 0.07 & 0.21 & 1.00 & 0.17 & 139 \\
\hline 8 & 335 & 29 & 0.21 & 0.30 & 0.20 & 0.36 & 67 \\
\hline 9.69 & 396 & 70 & 0.00 & 0.61 & -1.20 & 1.50 & 16 \\
\hline
\end{tabular}

Notes: The same as in Table 3 but for the total sample of blue and red clusters (bins centered on even values of radius, see text for details).

2 arcmin. Our results (for mass and mass-to-light ratios) for the isotropic case are given in Table 8. In this table for comparison we also provide estimates for mass and mass-to-light ratio obtained using X-ray methodology in the same bins. The errors for the estimates based on the X-rays reflect uncertainty in the temperature. In the calculation of the mass (and mass-to-light ratios) based on the GCs we used $\gamma=1.54$ which was derived using a least-squares fit applied to the data published by Dirsch et al. (2003) for all the clusters in the sample. The outermost point at
10 arcmin was taken. The data from the Table 8 are graphically presented in Fig. 6. The large box contains estimates for the total mass-to-light ratio (open and filled triangles are for the determination based on the X-ray technique assuming hydrostatic equilibrium (open triangles are for $\beta=0.35$ and filled triangles are for $\beta=0.50$ ) and open circles are for the estimate based on GCs assuming isotropy. In the estimates of the mass-to-light ratio based on the X-rays we take $T=1 \mathrm{keV}$ in the first bin, and $T=1.30 \mathrm{keV}$ in all the other bins. In a small inserted box we plot 
Table 7. Kinematics data for NGC 1399 for the total sample of blue and red clusters (part 3).

\begin{tabular}{|c|c|c|c|c|c|c|c|}
\hline $\begin{array}{c}\langle r\rangle \\
(\operatorname{arcmin}) \\
(1)\end{array}$ & $\begin{array}{c}\sigma_{\text {total3 }} \\
\left(\mathrm{km} \mathrm{s}^{-1}\right) \\
(2)\end{array}$ & $\begin{array}{c}\text { err_ } \sigma_{\text {total3 }} \\
\left(\mathrm{km} \mathrm{s}^{-1}\right) \\
(3)\end{array}$ & $s_{3 \text { total3 }}$ & err $\_s_{3 \text { total } 3}$ & $S_{4 \text { total3 }}$ & err $\_s_{4 \text { total3 }}$ & (8) \\
\hline 1.47 & 322 & 26 & -0.28 & 0.28 & 1.40 & 0.32 & 75 \\
\hline 3.40 & 362 & 30 & 0.69 & 0.28 & 2.40 & 0.32 & 75 \\
\hline 4.51 & 330 & 27 & -0.56 & 0.28 & 0.20 & 0.32 & 75 \\
\hline 5.68 & 329 & 27 & 0.42 & 0.28 & 1.40 & 0.32 & 76 \\
\hline 6.73 & 281 & 23 & -0.14 & 0.28 & 1.40 & 0.32 & 75 \\
\hline 8.81 & 352 & 30 & 0.07 & 0.30 & -0.80 & 0.35 & 68 \\
\hline
\end{tabular}

Notes: The same as in Table 3 but for the total sample of blue and red clusters. The number of clusters per bin is approximately 75 , see text for details.

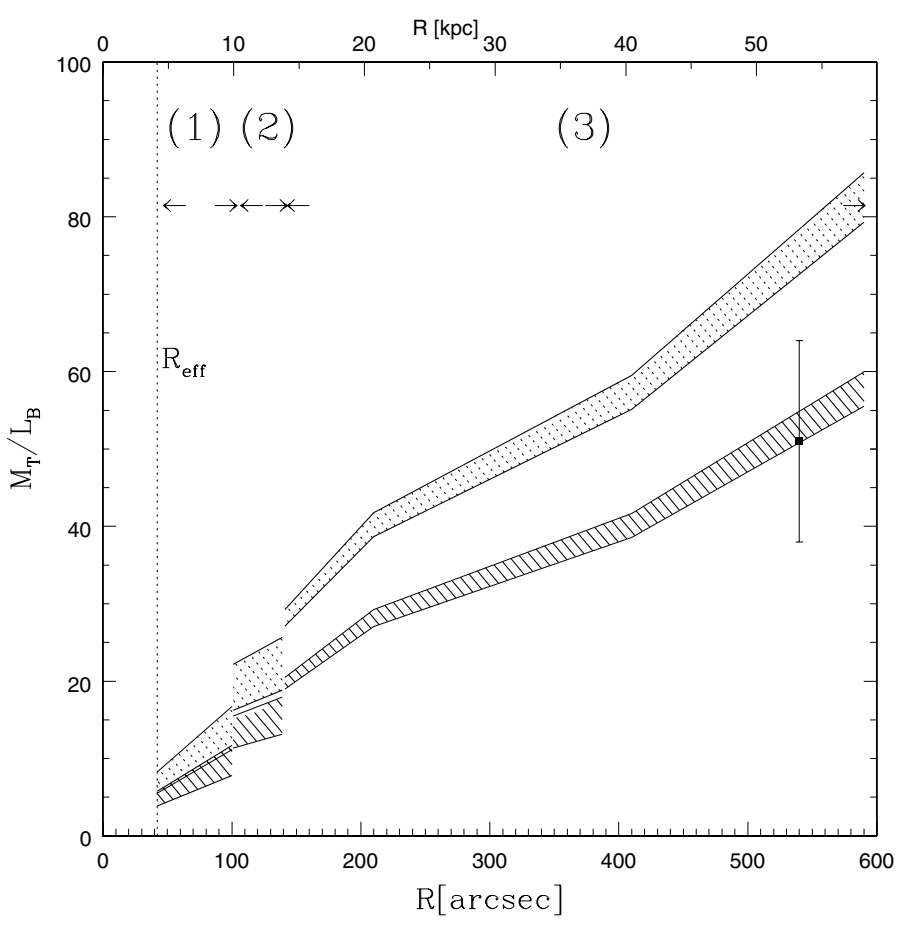

Fig. 5. Estimates of the total mass-to-light ratio in the $B$-band in the solar units using X-rays assuming hydrostatic equilibrium and isotropic velocity distribution presented with three shaded regions. Three different regions of the constant temperature were used beyond one effective radius: region (1) is between 40 and 100 arcsec, region (2) is between 100 and 140 arcsec and the region (3) is between 140 and 600 arcsec. (1): the shaded regions interior to 100 arcsec cover the temperatures between 0.61 and $0.92 \mathrm{keV}$ as estimated in Brown \& Bregman (1998); (2): shaded regions between 100 and 140 arcsec have for a lower value $T \sim 0.88 \mathrm{keV}$ and for an upper value $T \sim 1.2 \mathrm{keV}$ as estimated from the paper of Jones et al. (1997, their Fig. 3); (3): beyond 140 arcsec $T=1.30 \pm 0.05$ was used as estimated by Jones et al. (1997). With a filled square we presented the mass-to-light ratio found by Grillmair et al. (1994, $\left.M_{T} / L_{B}=51 \pm 13\right)$. Vertical dotted line is one effective radius. The regions shaded with dots are for the case for which $\beta=0.50$ (Paolillo et al. 2002) and the regions shaded with lines are for the case for which $\beta=0.35$ (Jones et al. 1997).

the estimates for the mass: the symbols have the same meaning as in the large box. In the inner regions (interior to $\sim 6$ arcmin) of NGC 1399 we have good agreement between the two techniques. At $\sim 6$ arcmin and beyond the estimate of the mass (and the mass-to-light ratio) based on $\beta=0.50$ starts to diverge from the estimate based on the GCs, whereas the estimate based on $\beta=0.35$ is consistent with it up to 8 arcmin. Note also that the estimate of Grillmair et al. (1994) is consistent with both results based on the GCs and X-rays $(\beta=0.35$ case).

The only point for which there seems to be a discrepancy between the X-ray and the GC estimates is the one at 10 arcmin: the $\mathrm{X}$-rays (with $\beta=0.35$ ) predict the mass-to-light ratio of $59 \pm 2$ whereas the estimate based on the GCs gives $46 \pm 8$. There may be two possible sources of this discrepancy: (i) errors given in the $\mathrm{X}$-ray case should be understood as minimal, because in these estimates we took into account only the uncertainty in temperature $(T=1.30 \pm 0.05)$. Note that Jones et al. (1997) at 10 arcmin calculated $M / L_{B}=55 \pm 17$ (scaled to the distance used in our paper; see their Fig. 7 - in our Fig. 6 we plotted the points at 3 and 10 arcmin based on their plot); note also that at $\sim 10$ arcmin their best fit for the temperature is $1.22 \mathrm{keV}$ which is somewhat lower than the value we took thus implying a lower mass-to-light ratio which is closer to that obtained using GC methodology; (ii) the discrepancy between the two results may be real and might be attributed to the effect of a contribution to the X-ray gas pressure by the ICM referred to above. Peng et al. (2004) also used the Evans et al. (2003) "tracer mass estimator" to calculate the mass-to-light ratio in NGC 5128. They found at $80 \mathrm{kpc}\left(\sim 15 R_{\mathrm{e}}\right)$ that $M / L_{B} \sim 13$ which suggests even smaller amounts of dark matter in the outer parts of this galaxy.

Different cosmological models and their predictions for the mass may in principle be tested. Richtler et al. (2004, references therein) tested among others, logarithmic potentials and Navarro et al. (1996, NFW) mass profiles. With the data which extend out to $\sim 10$ arcmin it is difficult to draw firm conclusions; the mass predicted by both NFW profile and the logarithmic potential is closer to the mass based on GCs and X-rays for which $\beta=0.35$ than to the X-rays for which $\beta=0.50: M \sim 2.15 \times 10^{12} M_{\odot}$ at $\sim 10$ arcmin (scaled to the distance used in our paper; see the large open pentagon in the small inserted box of Fig. 6).

We see in Fig. 6 that there is agreement between the following four different estimates of the total mass-to-light ratio: (i) our estimate based on the GCs; (ii) our estimate based on the X-rays (the case with $\beta=0.35$ ); (iii) the estimate based on the Grillmair et al. (1994) paper based on the GCs; and (iv) the estimate based on the NFW approach (this last one is given only in the small inserted box of Fig. 6).

\section{Discussion and conclusions}

In this paper we investigated the line-of-sight velocity distribution (LOSVD) calculated from the observations of GCs in the early-type galaxy NGC 1399. We have used recently published Dirsch et al. (2004) data from which we calculated velocity dispersions using both standard statistical procedures and the maximum likelihood approach. 


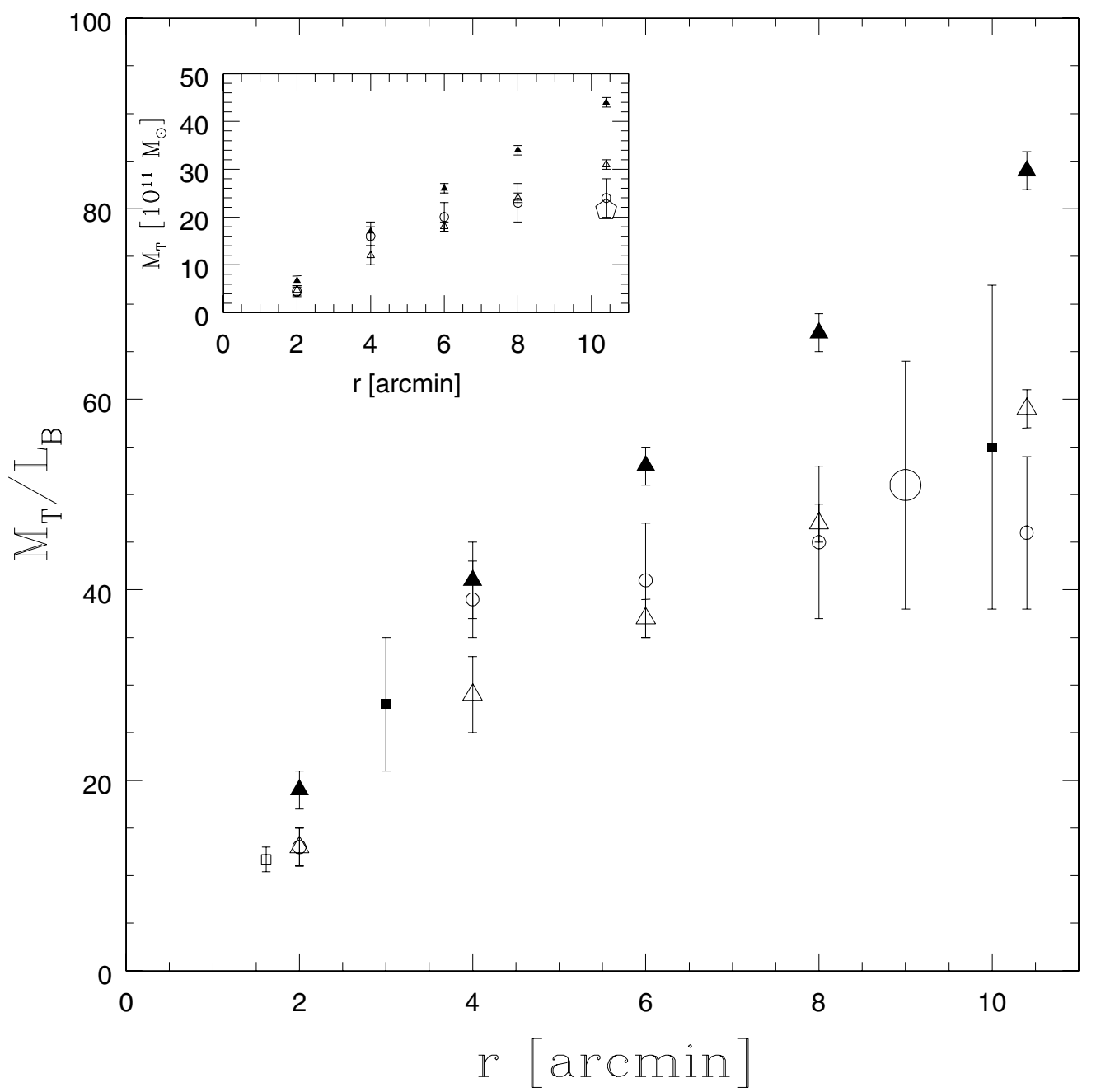

Fig. 6. Mass estimates using the GCs and X-ray methodology. Large box: estimates for the total mass-to-light ratio in the $B$-band in the solar units as a function of radius; the triangles are for the determination based on the X-ray technique assuming hydrostatic equilibrium (the open triangles for $\beta=0.35$ and the filled triangles for $\beta=0.50$ ), the open circles are for the estimate based on GCs assuming isotropy. The large open circle is the value obtained in the paper by Grillmair et al. (1994) scaled to the distance used in our paper (see text for details). The open square is the value obtained in the paper by Saglia et al. (2000) scaled to the distance used in our paper. The filled square is the value obtained in the paper by Jones et al. (1997) scaled to the distance used in our paper. The temperature is taken to be $1 \mathrm{keV}$ in the first bin and $1.30 \mathrm{keV}$ in all the other bins. Small inserted box: estimates of the total mass in units of $10^{11} M_{\odot}$ as a function of radius; the symbols have the same meaning as in the large box. The large open pentagon in the small box is for the estimates of Richtler et al. (2004) for the mass based on the Navarro et al. profile and the logarithmic potential.

Table 8. Mass estimates of NGC 1399 using globular clusters and X-rays.

\begin{tabular}{cccccccc}
\hline \hline Radius & $M_{\text {tot }}^{\mathrm{X}-\text { ray }, \beta=0.35}$ & $M^{\mathrm{X}-\text { ray }} / L_{B}$ & $M_{\text {tot }}^{\mathrm{X}-\text { ray }, \beta=0.50}$ & $M^{\mathrm{X}-\text { ray }} / L_{B}$ & $M^{\mathrm{GC}, \text { iso }}{ }_{\text {tot }}$ & $M^{\mathrm{GC}, \text { iso }} / L_{B}$ & $N$ \\
$\left(\begin{array}{c}\operatorname{arcmin}) \\
(1)\end{array}\right.$ & $10^{11} M_{\odot}$ & $M_{\odot} / L_{\odot}$ & $10^{11} M_{\odot}$ & $M_{\odot} / L_{\odot}$ & $10^{11} M_{\odot}$ & $M_{\odot} / L_{\odot}$ & \\
\hline$<2$ & $(2)$ & $(3)$ & $(4)$ & $(5)$ & $(6)$ & $(7)$ & $(8)$ \\
$<4$ & $4.7 \pm 1$ & $13 \pm 2$ & $6.7 \pm 1$ & $19 \pm 2$ & $4.4 \pm 1$ & $13 \pm 2$ & 14 \\
$<6$ & $12 \pm 2$ & $29 \pm 4$ & $17 \pm 2$ & $41 \pm 4$ & $16 \pm 2$ & $39 \pm 4$ & 157 \\
$<8$ & $24 \pm 1$ & $37 \pm 2$ & $26 \pm 1$ & $53 \pm 2$ & $20 \pm 3$ & $41 \pm 6$ & 283 \\
$<10.4$ & $31 \pm 1$ & $47 \pm 2$ & $34 \pm 1$ & $67 \pm 2$ & $23 \pm 4$ & $45 \pm 8$ & 413 \\
\hline 10.4 & $44 \pm 1$ & $84 \pm 2$ & $24 \pm 4$ & $46 \pm 8$ & 445 \\
\hline
\end{tabular}

Notes: Column (1): radius interior to which a given quantity is calculated; Col. (2): estimate of the total mass based on the X-ray methodology expressed in units of $10^{11} M_{\odot}$ for $\beta=0.35$ (the temperature was taken to be $1 \mathrm{keV}$ in the first bin and $1.30 \mathrm{keV}$ in all the other bins); Col. (3): mass-to-light ratio based on the X-ray methodology in the $B$-band in solar units for $\beta=0.35$; Col. (4): estimate of the total mass based on the X-ray methodology expressed in units of $10^{11} M_{\odot}$ for $\beta=0.50$ (the temperature was taken to be $1 \mathrm{keV}$ in the first bin and $1.30 \mathrm{keV}$ in all the other bins); Col. (5): mass-to-light ratio based on the X-ray methodology in the $B$-band in solar units for $\beta=0.50$; Col. (6): estimate of the total mass based on the GCs assuming isotropy (see text for details); Col. (7): mass-to-light ratio based on the GCs assuming isotropy in solar units (see text for details); Col. (8): the number of clusters in a given bin. 
Our conclusions are as follows.

(1) We show that the departures from the Gaussian distribution (given by the skewness and kurtosis parameters, $s_{3}$ and $s_{4}$, estimated from the radial velocities) are not large and that the estimates of the velocity dispersion calculated using standard statistical procedures and the maximum likelihood approach do not differ much. Using different binning we showed that slightly different trends in the velocity dispersion profile might be inferred.

(2) We find that inside $\sim 2.5 R_{\mathrm{e}}$ the mass is not dominated by dark matter (only for the case for which $\beta=0.50$ and $T \sim 0.92 \mathrm{keV}$ might one infer dark matter in this region). However, beyond $\sim 3 R_{\mathrm{e}}$ the high velocity dispersion and the $\mathrm{X}$-rays indicate the presence of dark matter. This is not inconsistent with our previous results (Samurović \& Danziger 2005) for 2 early-type galaxies (IC 1459, IC 3370) for which dark matter interior to $\sim 3 R_{\mathrm{e}}$ did not appear to dominate. Our estimates of the total mass (and mass-to-light ratio) based on the X-ray and GC methodology imply $\beta=0.35-$ this is in agreement with the value found in the paper by Jones et al. (1997). Beyond 8 arcmin there seems to be a discrepancy between the two methodologies: the GC data imply a smaller total mass than that obtained using X-rays; it is not clear whether this discrepancy lies within the uncertainties or not.

(3) Using the new "tracer mass estimator" of Evans et al. (2003) we find that the increase of the total mass of NGC 1399 beyond $\sim 4$ arcmin has a slow rise. At $\sim 10$ arcmin we calculated, for an isotropic case, $M / L_{B}=46 \pm 8$. In spite of the hint of the decreasing velocity dispersion in NGC 1399 (between $\sim 3$ and $\sim 7$ arcmin) the observations show evidence of dark matter in the outer parts of this galaxy. The procedure outlined in our paper provides a means of comparison between different calculations of the total mass of an earlytype galaxy without resorting to much more complex and computationally intensive superposition of orbits required in the Schwarzschild (1979) modelling.

(4) It is difficult to draw general conclusions regarding anisotropies in the outer parts of early-type galaxies at this point because the sample of galaxies is still too small and there are problems regarding the small number of observed clusters per galaxy. Judging from the sample of clusters used in this paper we did not find large departures from a Gaussian distribution. In a previous work (Samurović \& Danziger 2005) in the case of IC 1459 (beyond $\sim 2 R_{\mathrm{e}}$ ) and NGC 3379 (beyond $\sim 1.4 R_{\mathrm{e}}$ ) we found a hint of radial anisotropies. Dekel et al. (2005) using numerical simulations of disc-galaxy mergers recently found that for earlytype galaxies the stellar orbits in their outer parts are very elongated. Teodorescu et al. (2005) found that for PNe in the flattened early-type galaxy NGC 1344 the departures from a Gaussian distribution are small but they did not quantify them (see their Fig. 18).

(5) Given the fact that the full LOSVD profile of a given earlytype galaxy obtained from integrated stellar light is typically based on the integrated spectra of several million stars and the velocity dispersion based on GCs (and PNe) is based on at most several hundred objects, it is obvious that for more accurate kinematics of early-type galaxies at large radial distances an increased number of observed GCs (and PNe) is highly desirable.

Acknowledgements. We thank Francesca Matteucci for useful discussions. This research has made use of the NASA/IPAC Extragalactic Database (NED) which is operated by the Jet Propulsion Laboratory, California Institute of Technology, under contract with the National Aeronautics and Space Administration. S.S. was supported with grants from MIUR COFIN 1998 Prot. No. 9802909231_001, the "Regione Friuli-Venezia-Giulia" L.R. 3/98, CNAA Prot. No. 14/a and ASI Prot. No. I/R/043/02. S.S. expresses his gratitude to the TRIL (Training and Research in Italian Laboratories) programme of the Abdus Salam International Centre for Theoretical Physics. S.S. has been partially supported by the Ministry of Science and Environmental Protection of the Republic of Serbia through the project No. 1468, "Structure Kinematics, and Dynamics of the Milky Way". We thank the anonymous referee for the useful comments which helped to improve the manuscript.

\section{References}

Bahcall, N. A., Lubin, L. M., \& Dorman, V. 1995, ApJ, 447, L81 Bertin, G. 2000, Dynamics of galaxies (Cambridge: Cambridge Univ. Press) Brown, B. A., \& Bregman, J. N. 1998, ApJ, 495, L75

Cappellari, M., Bacon, R., Bureau, M., et al. 2006, MNRAS, 360, 1126 Ciotti, L., \& Pellegrini, S. 2004, MNRAS, 350, 609

Côté, P., McLaughlin, D. E., Cohen, J. G., \& Blakeslee, J. P. 2003, ApJ, 591, 850 De Rijcke, S., Prugniel, P., Simien, F., \& Dejonghe, H. 2006, MNRAS, accepted, preprint [arXiv: astro-ph/0603700]

Dekel, A., Stoehr, F., Mamon, G. A., Cox, T. J., \& Primack, J. R. 2005, preprint [arXiv:astro-ph/0501622]

de Vaucouleurs, G., de Vaucouleurs, A., Corwin, H. G. Jr., et al., Third Reference Catalogue of Bright Galaxies (New York: Springer-Verlag)

Dirsch, B., Richtler, T., Geisler, D., et al. 2003, AJ, 125, 1908

Dirsch, B., Richtler, T., Geisler, D., et al. 2004, AJ, 127, 2114

Evans, N. W., Wilkinson, M. I., Perrett, K. M., \& Bridges, T. J. 2003, ApJ, 583, 752

Forte, J. C., Faifer, F., \& Geisler, D. 2005, MNRAS, 357, 56

Fukazawa, Y., Botoya-Nonesa, J. G., Pu, J., Ohto, A., \& Kawano, N. 2006, ApJ, 636, 698

Grillmair, C. J., Freeman, K. C., Bicknell, G. V., et al. 1994, ApJ, 422, L9

Humphrey, P. J., Buote, D. A., Gastaldello, F., et al. 2006, ApJ, submitted, preprint [arXiv:astro-ph/0601301]

Jones, C., Stern, C., Forman, W., et al. 1997, ApJ, 482, 143

Kim, D.-W., \& Fabbiano, G. 1995, ApJ, 441, 182

Kronawitter, A., Saglia, R. P., Gerhard, O., \& Bender, R. 2000, A\&AS, 144, 53

Mathews, W. G., \& Brighenti, F. 2003, ARA\&A, 41, 191

Méndez, R. H., Riffeser, A., Kudritzki, R.-P., et al. 2001, ApJ, 563, 135

Merritt, D. 1997, AJ, 114, 228

Morrison, H., Flynn, C., \& Freeman, K. C. 1990, AJ, 100, 1191

Navarro, J. F., Frenk, C. S., \& White, S. D. M. 1996, ApJ, 462, 563

O’Sullivan, E., Ponman, T. J., \& Collins, R. S. 2003, MNRAS, 340, 1375

Paolillo, M., Fabbiano, G., Peres, G., \& Kim, D.-W. 2002, ApJ, 565, 883

Peng, E. W., Ford, H. C., \& Freeman, K. C. 2004, ApJ, 602, 705

Pryor, C., \& Meylan, G. 1993, in Structure and Dynamics of Globular Clusters, ed. S. G. Djorgovski, \& G. Meylan, ASP Conf. Ser., 50, 357

Richtler, T., Dirsch, B., Gebhardt, K., et al. 2004, AJ, 127, 2094

Romanowsky, A. J., Douglas, N. G., Arnaboldi, M., et al. 2003, Science, 5640, 1696

Saglia, R. P., Kronawitter, A., Gerhard, O., \& Bender, R. 2000, AJ, 119, 153

Samurović, S., \& Danziger, I. J. 2005, MNRAS, 363, 769

Schwarzschild, M. 1979, ApJ, 232, 236

Sivakoff, G. R., Sarazin, C. L., \& Carlin, J. L. 2004, ApJ, 617, 262

Teodorescu, A. M., Méndez, R. H., Saglia, R. P., et al. 2005, ApJ, 635, 290

Thomas, J., Saglia, R. P., Bender, R., et al. 2005, MNRAS, 360, 1355 